\title{
Stage II Adult Liver Cancer AJCC v7
}

National Cancer Institute

\section{Source}

National Cancer Institute. Stage II Adult Liver Cancer A/CC V7. NCI Thesaurus. Code C94776.

For hepatocellular carcinoma: Stage II includes: T2, N0, M0. T2: Solitary tumor with vascular invasion or multiple tumors none more than $5 \mathrm{~cm}$. N0: No regional lymph node metastasis. M0: No distant metastasis. For intrahepatic cholangiocarcinoma: Stage II includes: T2, N0, M0. T2: Solitary tumor with vascular invasion or multiple tumors, with or without vascular invasion. N0: No regional lymph node metastasis. M0: No distant metastasis. (from AJCC 7th Ed.) 